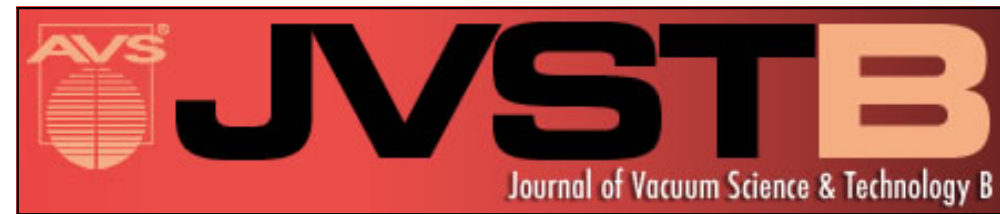

\title{
Binding energy, vapor pressure, and melting point of semiconductor nanoparticles
}

H. H. Farrell and C. D. Van Siclen

Citation: Journal of Vacuum Science \& Technology B 25, 1441 (2007); doi: 10.1116/1.2748415

View online: http://dx.doi.org/10.1116/1.2748415

View Table of Contents: http://scitation.aip.org/content/avs/journal/jvstb/25/4?ver=pdfcov

Published by the AVS: Science \& Technology of Materials, Interfaces, and Processing

\section{Articles you may be interested in}

Size and alloying induced changes in lattice constant, core, and valance band binding energy in Pd-Ag, Pd, and Ag nanoparticles: Effect of in-flight sintering temperature

J. Appl. Phys. 112, 014307 (2012); 10.1063/1.4731714

A sintered nanoparticle p-n junction observed by a Seebeck microscan

J. Appl. Phys. 111, 054320 (2012); 10.1063/1.3693609

Modeling pulsed-laser melting of embedded semiconductor nanoparticles

J. Appl. Phys. 110, 094307 (2011); 10.1063/1.3658265

Comparative study on size dependence of melting temperatures of pure metal and alloy nanoparticles Appl. Phys. Lett. 99, 013108 (2011); 10.1063/1.3607957

Size dependence of core and valence binding energies in Pd nanoparticles: Interplay of quantum confinement and coordination reduction

J. Appl. Phys. 104, 064308 (2008); 10.1063/1.2973682

\section{PIDEN}

Contact Hiden Analytical for further details: w www.HidenAnalytical.com E info@hiden.co.uk CLICK TO VIEW our product catalogue

\section{Instruments for Advanced Science}

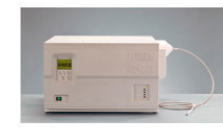

Gas Analysis

dynamic measurement of reaction gas streams catalysis and thermal analysis

molecular beam studies

, fermentation, environmental and ecological studies

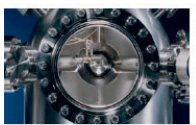

Surface Science UHVTPD

SIMS

end point detection in ion beam etch elemental imaging - surface mapoing

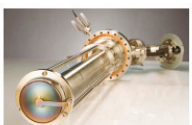

Plasma Diagnostics - plasma source characterization etch and deposition process reaction kinetic studies analysis of neutral and radical species

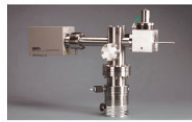

Vacuum Analysis partial pressure measurement and contro of process gases reactive sputter process control vacuum diagnostics vacuum coating process monitorin 


\title{
Binding energy, vapor pressure, and melting point of semiconductor nanoparticles
}

\author{
H. H. Farrell ${ }^{a)}$ and C. D. Van Siclen \\ Idaho National Laboratory, MS 2211, P.O. Box 1625, Idaho Falls, Idaho 83415
}

(Received 15 January 2007; accepted 6 April 2007; published 31 July 2007)

\begin{abstract}
Current models for the cohesive energy of nanoparticles generally predict a linear dependence on the inverse particle diameter for spherical clusters, or, equivalently, on the inverse of the cube root of the number of atoms in the cluster. Although this is generally true for metals, the authors find that for the group IV semiconductors, $\mathrm{C}, \mathrm{Si}$, and $\mathrm{Ge}$, this linear dependence does not hold. Instead, using first principles, density functional theory calculations to calculate the binding energy of these materials, they find a quadratic dependence on the inverse of the particle size. Similar results have also been obtained for the metallic group IV elements $\mathrm{Sn}$ and $\mathrm{Pb}$. This is in direct contradiction to current assumptions. Further, as a consequence of this quadratic behavior, the vapor pressure of semiconductor nanoparticles rises more slowly with decreasing size than would be expected. In addition, the melting point of these nanoparticles will experience less suppression than experienced by metal nanoparticles with comparable bulk binding energies. This nonlinearity also affects sintering or Ostwald ripening behavior of these nanoparticles as well as other physical properties that depend on the nanoparticle binding energy. The reason for this variation in size dependence involves the covalent nature of the bonding in semiconductors, and even in the "poor" metals. Therefore, it is expected that this result will hold for compound semiconductors as well as the elemental semiconductors. (C) 2007 American Vacuum Society. [DOI: 10.1116/1.2748415]
\end{abstract}

\section{BACKGROUND}

While the effect of nanoparticle or quantum dot size on a variety of physical properties, such as adsorption or emission frequency, melting point, etc., is well known, its effect on vapor pressure is less commonly considered. Kelvin's equation, which was derived to describe the vapor pressure of small droplets, predicts that the logarithm of the vapor pressure of such droplets is inversely proportional to the radius of the droplet. That is,

$$
\ln \left(P / P_{0}\right)=2 M \gamma / R T \rho r,
$$

where $P$ is the vapor pressure of the droplet, $P_{0}$ is the pressure from a planar surface, $M$ is the molecular weight, $\gamma$ the surface tension, $R$ the gas constant, $T$ the temperature, $\rho$ the density, and $r$ the radius. While the Kelvin equation is appropriate for macroscopic structures, particularly liquid droplets, it is less suitable for solid nanoparticles below about $10 \mathrm{~nm}$ in size. The reasons for this are several fold. One is that the surface tension itself becomes a size-dependent parameter in the lower end of this regime. Another is that, at least for solid particles large enough to display well developed facets, the surface energy is expected to vary with the index of the particular face under investigation.

Some of the current theoretical formalisms that consider the size dependence of quantities such as the melting point consider the problem in terms of the "cohesive energy" of the system rather than the surface tension. Arguably, for solid nanoparticles, the cohesive energy of the particle is a more useful parameter than surface tension. However, there is gen-

a)Electronic mail: helen.farrell@inl.gov erally an equivalence between the two different constructs. Both can also be used not only to determine vapor pressure over a particle but also to estimate its melting point, sintering rate, and a variety of other physical phenomena.

There currently exist several detailed theoretical models for determining the thermodynamic properties of solid nanoparticles based on either their cohesive energy or their surface tension. These formalisms include the liquid drop model $(\mathrm{LDM}),{ }^{1}$ the surface area difference (SAD) model, ${ }^{2}$ the bond order length (BOL) model, ${ }^{3}$ and the "thermodynamic" model. ${ }^{4}$ Several of these models consider the size dependence of nonspherical nanoparticles. As an interesting example of a high aspect ratio nonspherical case, Sun et al. use their BOL model to predict that the melting point of a monatomic chain of Au atoms will be suppressed by a factor of about 4.2 from the bulk value of 1337.32 to around $320 \mathrm{~K}{ }^{3}$

The other models also predict suppression of either the binding energy or the melting point as the size is diminished. Vanithakumari and Nanda have developed their LDM based on the coordination number of the surface atoms relative to that of the interior, bulk atoms. ${ }^{1}$ They assume that the total cohesive energy of a nanoparticle is equal to that of a bulk aggregate of the same volume of the nanoparticle minus the surface energy of the nanoparticle. They predict that the peratom cohesive energy for a spherical nanoparticle will be

$$
E_{b}=E_{b}^{\infty}-6 v_{0} \gamma_{n} / D
$$

where $E_{b}^{\infty}$ is the per-atom cohesive energy for the bulk material, $v_{0}$ is the atomic volume, $\gamma_{n}$ is the surface tension (which they consider to be size dependent below about 5 or $10 \mathrm{~nm}$ ), and $D$ is the diameter of the nanoparticle. 
The SAD model of Qi et al. takes surface effects into account by considering the areal atomic density of the planes or facets that bound the nanoparticle, which is assumed to have the bulk structure internally. ${ }^{2}$ It is expected to be applicable for nanoparticles in the $1-100 \mathrm{~nm}$ range. For a spherical nanoparticle, where all of the surfaces or interfaces have the same plane index, they find

$$
E_{b}=E_{b}^{\infty}\left[1-\left(3 p d_{h k l} / D\right)\right],
$$

where $p=\left(\gamma_{i} / \gamma_{0}\right)$ and $\gamma_{i}$ and $\gamma_{0}$ are the energies, per unit area at $0 \mathrm{~K}$, for the interface, or surface, and for the crystal, respectively. Here, $d=a / \sqrt{ }\left(h^{2}+k^{2}+l^{2}\right)$, with $a$ being the lattice parameter, and, again, $D$ is the diameter of the nanoparticle. For spherical nanoparticles, they also predict a parallel expression for the size dependence of the melting point as

$$
T_{m}=T_{m}^{\infty}\left[1-\left(3 p d_{h k l} / D\right)\right],
$$

where $T_{m}^{\infty}$ is the melting point of the bulk material.

Wautelet et al. have developed still another approach to describe the thermodynamic properties of both spherical and nonspherical nanoparticles. ${ }^{4}$ Based on their formalism, they expect that the melting point of a spherical nanoparticle will be given by

$$
T_{m}=T_{m}^{\infty}[1-(\alpha / D)],
$$

where $\alpha=6\left(\gamma_{c}-\gamma_{l}\right) / C$ and $\gamma_{c}$ and $\gamma_{l}$ are the surface tensions of the crystal and the liquid, respectively, and $C$ is the latent heat for melting. (They also note that a careful evaluation of $\alpha$ will take into account the change in density upon melting.)

As can be seen from Eqs. (2) to (4), these models all predict an inverse dependence on the diameter or radius of the nanoparticle for either or both the binding energy or the melting point. In fact, Vanithakumari and Nanda make the statement that "According to all theoretical models, the cohesive energy of free nanoparticles decreases linearly with the inverse of the particle size." While this is somewhat of a generalization, it is true that the LDM, SAD, and thermodynamic models all predict that the per-atom binding energy for spherical nanoparticle having $n^{3}=\mathrm{N}$ atoms all scales with size or $n$ as either

$$
E_{b n}=E_{b}^{\infty}[1-c / r]
$$

or

$$
E_{b n}=E_{b}^{\infty}\left[1-c^{\prime} / n\right],
$$

where $c$ and $c^{\prime}$ are materials-dependent constants, and $r$ is the radius of the cluster. (Note that for spherical nanoparticles, where the radius $r$ is equal to $n r_{0}$ and $r_{0}$ is the effective radius for one atom, Eqs. (5a) and (5b) are essentially equivalent.) It should also be noted that, for nonspherical configurations with high aspect ratios, such as nanowires and thin films, Qi et al., among others, have given a detailed description as how to modify Eq. (5a) and (5b) to accommodate shape effects. ${ }^{2}$

While these models agree in their general functionality for low aspect ratio clusters, they vary somewhat in the form for $c$ or $c^{\prime}$. To parse the relative validity of these models, a

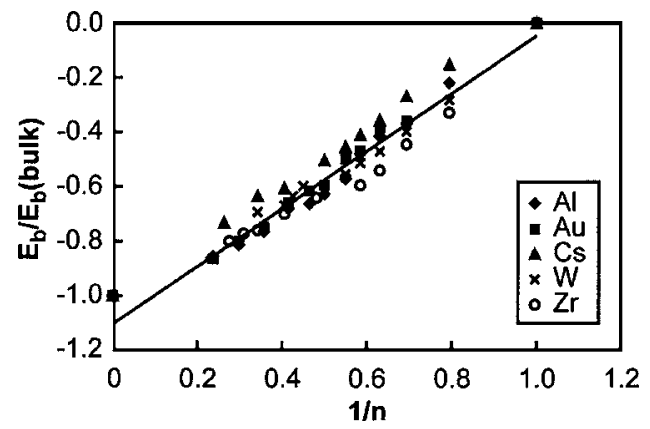

FIG. 1. Ratio of the per-atom binding energy to the bulk value is shown as a function of $1 / n$, where $n^{3}=N$ is the number of atoms in the cluster, for $\mathrm{Al}$, $\mathrm{Au}, \mathrm{Cs}, \mathrm{W}$, and $\mathrm{Zr}$. The straight line is the best fit to the Al data. The point on the far right corresponds to a single atom while that on the far left corresponds to bulk materials.

detailed comparison with a substantial body of experimental data is desirable. However, while a number of experimental studies do exist, ${ }^{5-7}$ there are often difficulties in interpreting the experimental results, particularly for very small particles. These difficulties can involve a number of factors including inadvertent oxidation, size changes due to sintering or sublimation, substrate effects, and artifacts or uncertainties introduced by the measurement technique.

Therefore, in order to supplement these experimental results, we set out to calculate the cohesive energy or bonding energy for a wide variety of simple, elemental materials. In this work, we focus on the group IV elements, C, Si, Ge, Sn, and $\mathrm{Pb}$. In a companion work, we studied a range of "good" metals, including those with the face-centered cubic, the body-centered cubic, and the hexagonal close packed structures, from various parts of the Periodic Table. ${ }^{8}$ For the metals studied in this companion work, we generally found that for the more stable clusters, a reasonably good agreement with Eq. (5b) was obtained. (The one exception to this trend was $\mathrm{Mg}$, which is arguably not a good metal.)

A normalized version of this dependence is shown in Fig. 1 for a subset of these metals that we studied. Here, we plot $E_{b} / E_{b}^{\infty}$ vs $1 / n$ for several metals including $\mathrm{Al}, \mathrm{Au}, \mathrm{Cs}, \mathrm{W}$, and $\mathrm{Zr}$. Similar results were also found for a variety of other metals. To a good approximation, we found that $E_{b}$ was linearly dependent on $1 / n$ at least for nanoparticles up to about $2 \mathrm{~nm}$. Somewhat more surprisingly, we found that $c^{\prime}$ is about unity in this size range. However, and rather more surprisingly, this linear dependence does not hold for the semiconductors, $\mathrm{C}, \mathrm{Si}$, and $\mathrm{Ge}$, nor does it hold for the "poor" metals $\mathrm{Sn}$ and $\mathrm{Pb}$. The details of this nonlinearity will be discussed below and are the motivation for this article. (For simplicity, we will use the term poor metals to denote one with a significant covalent, or directional, character. Conversely, good metals will denote those with predominantly nondirectional bonding.)

\section{MODELING}

In order to study these enthalpies of sublimation, we have performed first principles, density functional theory calculations on a variety of different clusters to obtain binding en- 
TABLE I. Comparison between experimental and calculated lattice parameters, bulk heat of formation, and diatomic molecule bond strength for the group IV elements.

\begin{tabular}{|c|c|c|c|c|c|c|}
\hline & \multicolumn{2}{|c|}{$a(\AA)$} & \multicolumn{2}{|c|}{$E_{b}^{\infty}(\mathrm{eV})$} & \multicolumn{2}{|c|}{$E_{d}(\mathrm{eV})$} \\
\hline & Expt. & Calc. & Expt. & Calc. & Expt. & Calc. \\
\hline $\mathrm{C}$ & 3.567 & 3.533 & -7.371 & -7.615 & $-6.29 \pm 0.22$ & -6.672 \\
\hline $\mathrm{Si}$ & 5.431 & 5.375 & -4.677 & -4.363 & $-3.39 \pm 0.10$ & 3.968 \\
\hline $\mathrm{Ge}$ & 5.658 & 5.548 & -3.874 & -3.763 & $-2.73 \pm 0.07$ & 3.232 \\
\hline$\alpha-\mathrm{Sn}$ & 6.412 & 6.383 & -3.13 & -3.017 & $-1.94 \pm 0.17$ & 2.727 \\
\hline $\mathrm{Pb}$ & 4.939 & 5.032 & -2.028 & -2.363 & $-0.90 \pm 0.01$ & -1.778 \\
\hline
\end{tabular}

ergies and $\Delta H_{s}$ in the generalized gradient approximation (GGA), including spin polarization. The modeling program that we used for calculating clusters was the commercially available $\mathrm{DMol}^{4.0}$ or $\mathrm{DMol}^{4.1}$ codes. ${ }^{9,10}$ Unless otherwise noted, with this program we used an all electron, relativistic, real-space numerical basis set ("DND"). In the $\mathrm{DMol}^{4.0}$ and $\mathrm{DMol}^{4.1}$ codes, the GGA is implemented with the PerdewWang-91 (Ref. 11) parameterization of the correlation energy. For the bulk reference materials, we first used the companion CASTEP program to calculate geometries, and then used the DMol programs to calculate binding energies.

As a check on the general validity of our calculations, we show a comparison between our calculated values and the tabulated experimental values for the bulk lattice parameters, ${ }^{12}$ bulk heat of formation, ${ }^{13}$ and the bond strengths for the diatomic molecules ${ }^{14}$ in Table I. It should be noted that all of the calculated values are essentially for $0 \mathrm{~K}$, while the experimental values for the lattice parameters and diatomic molecules are for room temperature. Therefore, in the case of the lattice parameters, it is to be expected that the calculated values are slightly smaller than the experimental values. (The well known tendency for density functional theory calculations to "overbind" is also a contributing factor here.) Even so, the calculated bulk lattice parameters all agree to within $2 \%$, with a rms deviation of $1.54 \%$. This comparison primarily checks the CASTEP formalism.

In the case of the bulk enthalpies, we used the negative of the $0 \mathrm{~K}$ value for the standard heat of formation for a single, neutral atom. (By convention, the bulk is taken as the standard state for all of these elements.) Note that the calculated value is for "gray," or $\alpha$-Sn, while the tabulated value is for "white," or $\beta$-Sn, which is the form stable at room temperature. Here, excluding $\mathrm{Sn}$, the calculated values agree with the experimental values to within $17 \%$ for $\mathrm{Pb}$ and to within $2 \%-7 \%$ for the lighter elements. In this comparison, we use both the CASTEP formulation and the localized $\mathrm{DMol}^{4}$ formulation, so that this is a good check on both computational methods.

The experimental values for the diatomic molecule bond strengths are taken from a compilation of different experimental values. ${ }^{14}$ These values are generally measured either spectroscopically or by mass spectrometer analysis of hot gases effusing from a Knudsen cell. The reported values have all been corrected to room temperature with varying degrees of approximation. ${ }^{14}$ As can be seen in Table I, while the detailed agreement is not as good for the diatomic bond strengths as it is, for example, for the lattice parameters, the same general trend occurs for both the calculated and experimental values.

A number of authors have carefully studied the variations in binding energy as a function of the detailed geometry or configuration of nanoparticles with a given number of atoms. These include, for example, the work by Xiao and Wang on Pt clusters ${ }^{15}$ and by Chuang et al. on Sn clusters. ${ }^{16}$ While we generally sought the most stable configuration for a given number of atoms, it is not our purpose to make a careful study of the variation in binding energy as a function of detailed configuration, and we note this effect only briefly in the next section.

\section{RESULTS}

Carbon shows the greatest flexibility in forming different types of strong, directional bonds with varying hybridizations; a fact that makes it the key element in the multitudinous organic compounds necessary to life. In Fig. 2 we show the per-atom binding energy for a wide variety of carbon clusters including linear arrangements, nanotubes, and the $\mathrm{C}_{60}$ "buckeyball." Also shown are the binding energies for graphite, diamond, and two types [(5-5) and (12-0)] of nanotubes of infinite length. At $0 \mathrm{~K}$, graphite is the most stable bulk allotrope, having a binding energy of $-7.7195 \mathrm{eV}$ rela-

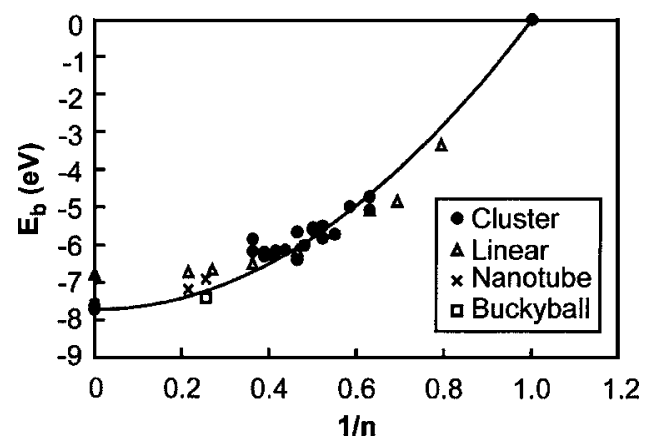

FIG. 2. Per-atom binding energy is shown as a function of $1 / n$, where $n$ is the cube root of the number of atoms in a cluster, for a variety of different types of $\mathrm{C}$ structures. These calculations were done with the GGA method. The solid line corresponds to a quadratic $\left[1-(1 / n)^{2}\right]$ dependence. 
tive to a free, isolated $\mathrm{C}$ atom. This is $0.104 \mathrm{eV}$ lower than the calculated binding energy for diamond at $0 \mathrm{~K}$.

The two infinite nanotubes are slightly less stable, having per-atom binding energies of -7.656 and $-7.570 \mathrm{eV}$ for the (12-0) and (5-5) configurations, respectively. Not surprisingly, among the near-spherical clusters for which we performed calculations, the $\mathrm{C}_{60}$ buckeyball was the most stable, having a binding energy of $-7.399 \mathrm{eV}$. As can be seen from Fig. 2, nanotubes in this mass range were also relatively stable with the 100 atom (5-5) structure having a binding energy of $-7.176 \mathrm{eV}$, and the 60 atom (5-5) structure having $-6.1905 \mathrm{eV}$.

While it is not the purpose of this work to explore the detailed differences among different bonding configurations, it is of interest to note in passing that, at least on the scale of the bulk binding energy, the per-atom binding energy is rather similar for a variety of different cluster configurations in the size range between several atoms and several hundred atoms. This is true even in the extreme of linear chains of carbon atoms. In fact, for an infinite chain of carbon atoms (Fig. 2), the binding energy is $-6.771 \mathrm{eV}$, which is about $88 \%$ of the bulk value. This can be compared to the case for a linear chain of $\mathrm{Au}$ atoms where the suppression is reported to be considerably larger (e.g., to less than $25 \%$ of the bulk value). ${ }^{3}$

Perhaps the most important feature of Fig. 2 is the nonlinear dependence of the per-atom binding energy on $1 / n$, the cube root of the total number of atoms in a cluster in this mass range. For the near spherical nanoparticles, this is equivalent to a nonlinear dependence on the inverse of the radius or diameter of the particles. This behavior is in marked contrast to that which we observed for the metallic clusters shown in Fig. 1 and discussed in Sec. I. Further, it is different from the dependence predicted by the current theoretical models. ${ }^{1,2,4}$ In fact, for these group IV elements, rather than being linear in $1 / n, E_{b}$ is better fitted by a quadratic $(1 / n)^{2}$ functionality, as shown by the solid curve in Fig. 2 . Therefore, for $\mathrm{C}$ (and, as we shall see, for $\mathrm{Si}, \mathrm{Ge}, \mathrm{Sn}$, and $\mathrm{Pb}$ as well) we can write

$$
E_{b n}=E_{b}^{\infty}\left[1-(1 / n)^{2}\right]
$$

Though some scatter exists in our calculated values for $E_{b}$, as can be seen in Fig. 2, this quadratic functionality is a reasonable approximation for the more stable clusters. Given that, at sufficiently high temperature, if the clusters do not sinter or sublime, they will eventually evolve into the most stable configurations, we can use this $(1 / n)^{2}$ functionality to describe the behavior of the binding energy of an ensemble of nanoparticles. This gives us a powerful predictive tool not only for individual particles but also for physically realistic distributions of nanoparticles.

As a final point before turning to our calculations for $\mathrm{Si}$ and the other group IV elements, we would like to note that, as an additional check on the general validity of our computations, we also performed calculations on a variety of clusters using both relativistic and nonrelativistic (not shown) basis sets. In both cases, to a good approximation, the qua-

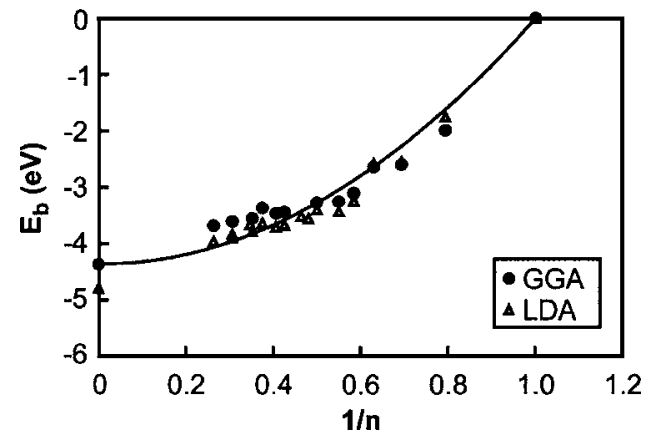

FIG. 3. Per-atom binding energy is shown as a function of $1 / n$ for a variety of different types of Si clusters. These calculations were done with both the LDA and the GGA method. The solid line corresponds to a quadratic dependence.

dratic dependence of $E_{b}$ on $1 / n$ was observed. We take this result as one indication that this quadratic behavior is not an artifact of our computational methods. In fact, the underlying physics is rather simple. Carbon, and to a lesser extent the other group IV elements, has directional bonds and the ability to form a variety of differently hybridized states.

Therefore, decreasing the number of nearest neighbors by forming, for example, a surface does not decrease the binding energy in proportion to the decrease in the number of nearest neighbors. This is because the $\mathrm{C}$ will rehybridize from its bulk configuration to form additional bonds to the remaining neighboring atoms. In the extreme of an isolated $\mathrm{C}_{2}$ dimer molecule, there will actually be roughly three bonds (one $\sigma$ and two $\pi$ ) between the $\mathrm{C}$ atoms rather than just one bond. As most of the current theories of cluster binding energies either explicitly or tacitly assume that the binding energy is proportional to the number of nearest neighbors (an assumption that works more or less for good metals), they underestimate the binding energies for the group IV elements and most likely for compound semiconductors as well.

Continuing with our examination of the other group IV elements, in Fig. 3, we show the per-atom binding energy of $\mathrm{Si}$ as a function of $1 / n$. As can be seen, the general behavior is similar to that for carbon. Here again, $E_{b}$ for the most stable clusters is fitted to a reasonable approximation by a $(1 / n)^{2}$ functionality, as shown by the solid line. Therefore, we expect Eq. (6) to be a reasonable approximation for $E_{b}$ for $\mathrm{Si}$ as well as for $\mathrm{C}$.

Similar comments can be made about the binding energy of Ge clusters, as shown in Fig. 4. Again, to a good approximation, the most stable clusters have binding energies that are fitted by a $(1 / n)^{2}$ rather than a $1 / n$ dependence, and Eq. (6) is expected to hold. As a second check on our computations, we calculated $E_{b}$ for both $\mathrm{Si}$ and Ge using two different methods, the local density approximation (LDA) and the GGA. As can be seen here and in Fig. 3, both methods produced a nonlinear dependence of $E_{b}$ on $1 / n$, though it is slightly less pronounced for the presumably less reliable LDA method. 


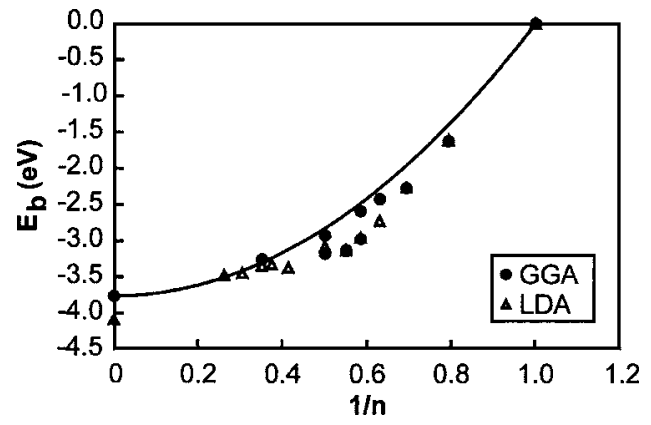

FIG. 4. Per-atom binding energy is shown as a function of $1 / n$ for a variety of different types of Ge clusters. These calculations were done with both the LDA and the GGA method. The solid line corresponds to a quadratic dependence.

These general trends continue for the more metallic group IV elements, Sn (Fig. 5) and Pb (Fig. 6). As before, in the mass range that we investigated, both LDA and GGA calculations were reasonably approximated by a quadratic $(1 / n)^{2}$ behavior, though the LDA values diverge from the more accurate GGA values for these heavier elements. For simplicity, only the GGA values are shown in Figs. 5 and 6.

Our cluster calculations indicate that for small, low aspect ratio clusters of the elemental semiconductors and the more metallic group IV elements a significantly different behavior is to be expected in those physical properties that are strongly dependent on $E_{b}$, such as melting point, vapor pressure, sintering behavior, solubility, etc., than is predicted by the commonly accepted current theories. Specifically, in the case of the melting point, we expect a size-dependent behavior that can be approximated by

$$
T_{m}=T_{m}^{\infty}\left[1-(1 / n)^{2}\right]
$$

or

$$
T_{m}=T_{m}^{\infty}\left[1-\left(r_{0} / r\right)^{2}\right]
$$

rather than by Eq. (4), for example. ${ }^{17,18}$ This means that the melting point of clusters of these materials will be higher than expected on the basis of a linear dependence of $E_{b}$ on $1 / n$. As this behavior is undoubtedly due to the covalent or directional nature of the bonding in these materials, it is to be expected that similar behavior will occur for compound

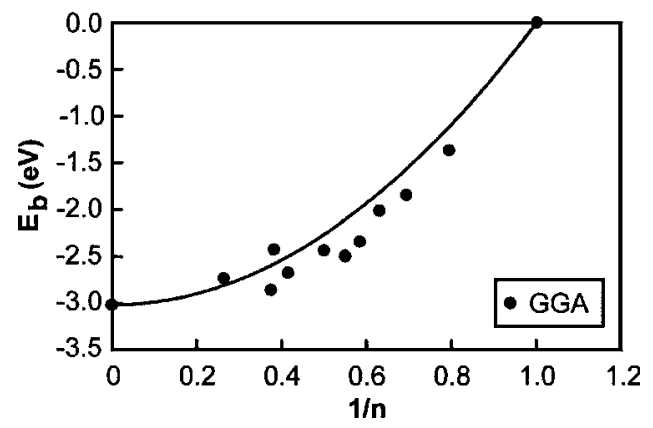

FIG. 5. Per-atom binding energy is shown as a function of $1 / n$ for a variety of different types of $\mathrm{Sn}$ clusters. Only the GGA calculations are shown in this figure. The solid line corresponds to a quadratic dependence.

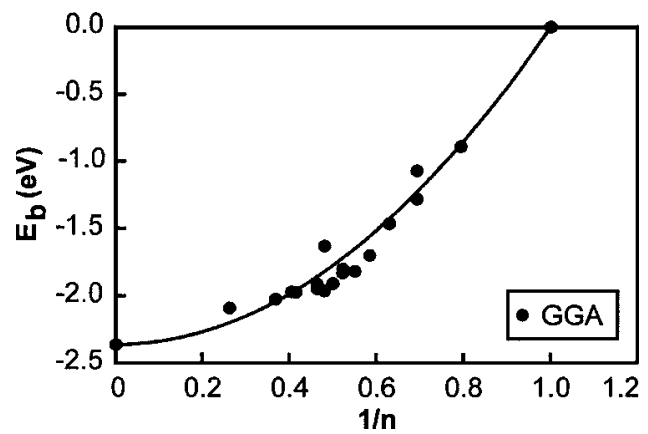

FIG. 6. Per-atom binding energy is shown as a function of $1 / n$ for a variety of different types of $\mathrm{Pb}$ clusters. Only the GGA calculations are shown in this figure. The solid line corresponds to a quadratic dependence.

semiconductors as well. Good experimental evidence will be needed to verify these predictions. As noted above, obtaining experimental evidence on small (e.g., $<2$ or $3 \mathrm{~nm}$ ) nanoparticles is a nontrivial process, but we hope that this will be taken as a challenge by first class surface scientists. [A particular complication for semiconductors is that, while Eqs. (6) and (7) have been derived for isolated nanoparticles, substrate effects, particularly if the nanoparticles are strongly epitaxied, will significantly influence experimental results.]

The vapor pressure of nanoparticles is also an extremely important physical property, though one less frequently considered than the melting point. We assume that the vapor pressure is given by an expression like

$$
P=P_{0} \exp \left(-\Delta H_{s} / k T\right),
$$

where $P_{0}$ is a prefactor that is only slowly temperature dependent, and $\Delta H_{s}$ is the enthalpy of sublimation. A careful examination of the statistical mechanical derivation of the vapor pressure shows that the term $P_{0}$ is derived from the properties of the gas phase rather than from those of the solid. Therefore, $P_{0}$ should be independent of the form of the solid, and we can use the same form and values as determined from bulk materials. For the enthalpy of sublimation, to a good approximation, we can neglect entropy effects and equate $\Delta H_{s}$ with $E_{b}$ as

$$
\Delta H_{s}=E_{b n}=E_{b}^{\infty}\left[1-(1 / n)^{2}\right] .
$$

As with the melting point, the vapor pressure of these group IV materials will deviate from the bulk values to a lesser extent than would be expected if $E_{b}$ was linearly dependent on $1 / n$. However, it should be noted that as $\Delta H_{s}$ appears in an exponent in Eq. (8), the size dependence of the vapor pressure of nanoparticles is very strong, even for the group IV elements and other semiconductors, and vapor pressures that are orders of magnitude larger than that of the bulk material may occur, particularly at elevated temperatures.

A third area of physical behavior that will be strongly affected by the size dependence of $E_{b}$ is that of sintering or Ostwald ripening. Following the classic work of Wynblatt and co-worker, ${ }^{19,20}$ we can write the time rate of change of a nanoparticle radius as 


$$
\begin{aligned}
d r / d t= & \left\{K_{1} /\left[r^{2} \ln (L / r \sin \theta)\right]\right\}\left\{\exp \left(\Delta \mu^{*} / k T\right)\right. \\
& -\exp (\Delta \mu / k T)\},
\end{aligned}
$$

where

$$
\Delta \mu=E_{b}^{\infty}-E_{b}^{\infty}\left[1-1 / n^{2}\right]=E_{b}^{\infty} / n^{2}
$$

and

$$
K_{1}=a^{2} \nu_{p} \Omega c_{s}^{\mathrm{eq}} \exp \left[-\left(H_{p s}+H_{m}^{s}\right) / k T\right] / 2 \alpha_{1} .
$$

Here, $a$ is the interatomic spacing, $\Omega$ is the atomic volume, $\nu_{p}$ is the vibrational frequency of a adatom on the nanoparticle, $c_{s}^{\mathrm{eq}}$ is the concentration of adatoms on the substrate support surface, $\alpha_{1}$ is a geometric factor which has the value of 0.5 when the nanoparticles are hemispherical and make an angle of $90^{\circ}$ with the substrate surface, ${ }^{19,20}$ and $\mu^{*}$ is the equilibrium mean binding energy at infinite time.

Also in Eq. (12), the quantity $H_{p s}$ is equal to $H_{p}-H_{s}$ where the former is the adsorption energy of an adatom on a nanoparticle and the latter is the adsorption energy on the support. $H_{p}$ is actually the previously determined $E_{b n}$ $=E_{b}^{\infty}\left[1-(1 / n)^{2}\right]$. The quantity $H_{m}^{s}$ is the activation energy for diffusion of an adatom on the substrate surface.

To better understand the differences in sintering behavior between nanoparticles with directional bonding and metallic bonding, let us focus on the term in brackets in Eq. (10), $\left\{\exp \left(\Delta \mu^{*} / k T\right)-\exp (\Delta \mu / k T)\right\}$. First, let us consider the case of a directionally bonded material. For $\mathrm{Si}$, for example, $E_{b}^{\infty}$ is on the order of $4.3 \mathrm{eV}$ such that for a temperature of $525^{\circ} \mathrm{C}$ (e.g., $873.16 \mathrm{~K}), E_{b}^{\infty} / k T$ is 62.52 . Therefore, if the equilibrium radius of the nanoparticles is $\geqslant 10 \mathrm{~nm}$, the first term, $\exp \left(\Delta \mu^{*} / k T\right)$, will be on the order of unity. (Here, we have taken the atom volume of $\mathrm{Si}$ to be $20.02 \AA^{3}$, with a corresponding radius of $1.68 \AA$, such that for $r^{*} \geqslant 10$, we will have $n \geqslant 60$.) However, for very small nanoparticle, $n$ will be much smaller, and the second term will be very much larger. For example, for hemispherical nanoparticles with $r$ $\approx 0.635 \mathrm{~nm}, n=3$, and the second term, $\exp (\Delta \mu / k T)$, will be $1.04 \times 10^{3}$. As this term is much larger than the first term (which is on the order of unity), it will dominate the expression in brackets in Eq. (10). Its negative sign indicates that these small nanoparticles are shrinking, or being consumed, during the formation of larger particles through the ripening process.

Now let us consider the case of a hypothetical metal with the same binding energy and atom size as Si. Here, $\Delta \mu$ $=E_{b}^{\infty}-E_{b}^{\infty}[1-1 / n]=E_{b}^{\infty} / n$ rather than being inversely dependent on $n^{2}$ as was the case for $\mathrm{Si}$. As a consequence, $\exp \left(\Delta \mu^{*} / k T\right)$ will be on the order of 10 , and $\exp (\Delta \mu / k T)$ will be on the order of $1.12 \times 10^{9}$. Note that the second term will still dominate and the nanoparticles of this size will still shrink. However, the rate at which they will do so will be much larger than for the case of Si. Therefore, the metallic material will undergo Ostwald ripening much faster than will the directionally bonded material. Conversely, semiconductors will sinter much more slowly than will metallic materials with similar binding energies under similar conditions.
For larger particles, the difference between the metallically bonded case and the directionally bonded case will decrease, but the same order will hold.

This is, of course, a much more complicated process than either sublimation or melting (though melting, per se, has a number of potential complications that we have not considered in this article ${ }^{6,16}$ ) and requires the calculation or determination of many more parameters than $E_{b}$. Despite these complications, the strong exponential dependence shown in Eqs. (10) and (11) and for $H_{p}$ shows that this process is significantly controlled by the size dependence of the binding energy and so will be markedly different for semiconductors and poor metals than for good metals.

While other physical phenomena are also dependent on the per-atom binding energy, these three examples should be adequate to illustrate the fact that the approximately quadratic dependence of $E_{b}$ on $1 / n$ for the group IV elements (and probably for compound semiconductors as well) should have a profound influence on the behavior of nanoparticles of these materials that would be incorrectly estimated by using the currently accepted inverse size dependence. Again, decisive experimental evidence is badly needed to verify or invalidate our theoretical results.

\section{CONCLUSION}

Current theoretical models describing the binding energy of nanoparticles based on the assumption that the binding energy is proportional to the number of nearest neighbors are not applicable to group IV elements. For low aspect ratio clusters, the prediction that the binding energy is inversely proportional to the size of the particle (or to $n$, the cube root of the number of atoms in a particle) does not hold for these materials. Instead, for small nanoparticles, an approximately quadratic functionality occurs such that the binding energy is inversely proportional to the square of the particle size (or $\left.n^{2}\right)$.

Similarly, the melting point of such particles will also have a $(1 / n)^{2}$ dependence, in contradiction to current theories. The vapor pressure of these materials will also be closer to the bulk value than would be expected by a $1 / n$ dependence of the sublimation energy, and other physical phenomena, such as sintering, solubility, etc., will also be strongly affected.

The physics of the situation is simple. Unlike most metals, where the binding energy is a function of the number of nearest neighbors in a cluster, the group IV elements have a strong degree of covalent, or directional, bonding. Though the trend diminishes as we go down the Periodic Table, these bonds can rehybridize, to varying degrees, to compensate for the loss of nearest neighbors at a surface. Therefore, the degree of bonding of surface atoms of these elements is higher than the simple number of nearest neighbors would predict. As this is a property associated with covalent bonding, it is expected that compound semiconductors, in addition to the elemental semiconductors, will also show this nonlinear dependence on $1 / n$. 


\section{ACKNOWLEDGMENT}

This research was supported in part by the U.S. Department of Energy, Office of Basic Energy Sciences, Division of Materials Sciences, under Contract No. DE-AC0799ID13727.

${ }^{1}$ See, for example, S. C. Vanithakumari and K. K. Nanda, J. Phys. Chem. B 110, 1033 (2006), and references therein.

${ }^{2}$ See, for example, W. H. Qi, M. P. Wang, M. Zhou, and W. Y. Hu, J. Phys. D 38, 1429 (2005), and references therein.

${ }^{3}$ See, for example, Chang Q. Sun, H. L. Bai, S. Li, B. K. Tay, C. Li, T. P. Chen, and E. Y. Jiang, J. Phys. Chem. B 108, 2162 (2004), and references therein.

${ }^{4}$ See, for example, M. Wautelet, J. P. Dauchot, and M. Hecq, J. Phys.: Condens. Matter 15, 3651 (2003), and references therein.

${ }^{5}$ See, for example, A. P. Alivisatos, J. Phys. Chem. 100, 13226 (1996), and references therein.
${ }^{6}$ K. F. Peters, J. B. Cohen, and Y.-W. Chung, Phys. Rev. B 57, 13430 (1998).

${ }^{7}$ M. Zhang et al., Phys. Rev. B 62, 10548 (2000).

${ }^{8}$ H. H. Farrell, C. D. Van Siclen, L. M. Petkovic, D. M. Ginosar, P. J. Pinhero, and H. W. Rollins (unpublished).

${ }^{9}$ B. Delley, J. Chem. Phys. 92, 508 (1990).

${ }^{10}$ B. Delley, J. Chem. Phys. 113, 7756 (2000)

${ }^{11}$ J. P. Perdew and Y. Wang, Phys. Rev. B 45, 13244 (1992).

${ }^{12}$ Handbook of Chemistry and Physics, 76th ed., edited by D. R. Lide (CRC, New York, NY, 1995), pp. 12-96.

${ }^{13}$ Handbook of Chemistry and Physics (Ref. 12), pp. 5-1-5-3.

${ }^{14}$ Handbook of Chemistry and Physics (Ref. 12), pp. 9-52-9-56.

${ }^{15}$ L. Xiao and L. C. Wang, J. Phys. Chem. A 108, 8605 (2004).

${ }^{16}$ F. C. Chuang, C. Z. Wang, S. Ogut, J. R. Chelikowsky, and K. M. Ho, Phys. Rev. B 69, 165408 (2004).

${ }^{17}$ J. H. Rose, J. Ferrante, and J. R. Smith, Phys. Rev. Lett. 47, 675 (1981).

${ }^{18}$ J. H. Rose, J. Ferrante, and J. R. Smith, Phys. Rev. B 25, 1419 (1982).

${ }^{19}$ P. Wynblatt and N. A. Gjostein, Acta Metall. 24, 1165 (1975).

${ }^{20}$ P. Wynblatt, Acta Metall. 24, 1175 (1975). 